\title{
Concept and empirical evaluation of a new curriculum to teach electricity with a focus on voltage
}

\author{
Jan-Philipp Burde ${ }^{1}$, and Thomas Wilhelm ${ }^{1}$ \\ ${ }^{1}$ Department of Physics Education Research, Goethe University, Max-von-Laue-Str.1, Frankfurt a.M., Hesse, Germany
}

\begin{abstract}
Effective reasoning about electric circuits requires a solid understanding of voltage and potential. However, most students fail to correctly analyze electric circuits as they tend to reason exclusively with current and resistance. To address these difficulties, a new curriculum based on the electron gas model was developed and empirically evaluated in a study with 790 students from Frankfurt, Germany. The study follows a pretest-posttest-control-group design and uses a recognized two-tier diagnostic test instrument, which also allows evaluating common alternative conceptions. The results of the diagnostic assessment are very promising; students taught according to the new curriculum not only develop a significantly better conceptual understanding of voltage, but also show achievement gains of about twice that of their traditionally taught peers.
\end{abstract}

Keywords: electric circuits, junior high school, curriculum, electric potential, voltage, electron gas model

\section{INTRODUCTION}

Despite many years of science education, students often do not develop the ability to reason qualitatively about electric circuits. Instead, they tend to resort to algorithmic manipulations when analyzing simple circuits as the mathematical introduction of Ohm's law often precedes a robust conceptual understanding of its underlying physical quantities [1]. However, even when considerable time is spent on developing a qualitative understanding of electric circuits, research on students' conceptual difficulties in electricity shows that voltage and electric potential typically remain unlearned even after instruction [2]. A particularly widespread alternative conception in this context is that voltage is a property or a component of an electric current rather than an independent physical quantity that refers to a difference in electric potential. Not realizing the importance of voltage as the cause of the flow of current, students tend to reason exclusively with current and resistance when analyzing electric circuits [3].

Even though these two concepts may appear to be more concrete and intuitive than voltage at first sight, a robust conceptual understanding of voltage as a difference in electric potential is key to effective analysis of electric circuits. Furthermore, the exclusive reasoning with current and resistance often contributes to the emergence of several other alternative conceptions such as sequential reasoning [4]. Cohen, Eylon and Ganiel [1] have hypothesized that the electric current dominates students' thinking about electric circuits because the concept of current is introduced before potential difference in almost every introductory course on electricity. In this context, they argue that "first impressions are strong and may impede a later, more rigorous, study of electricity" [pg. 411] and ask whether a possible remedy may lie in a curriculum that not only "[...] clearly spells out the relation of cause and effect between pd [potential difference] and current" [pg. 411], but also introduces the concept of potential difference first. Moreover, they suggest an analogy in which the battery exerts a certain 'pressure' on the electric charges in the wire. While the latter idea was picked up in the CASTLE curriculum [5] in the form of an air pressure analogy, voltage was still introduced after the electric current. Nonetheless, Steinberg \& Wainwright [6] report that students taught according to their curriculum show significantly larger achievement gains than traditionally taught students and see a major factor for the curriculum's effectiveness in its underlying highly intuitive yet powerful air pressure analogy. Unfortunately, the results of a statistical study on the effectiveness of the CASTLE curriculum have never been published.

What has been missing to date is the empirical evaluation of a curriculum that is based on the air pressure analogy but which introduces the concept of potential difference before electric current. In this article, therefore, we briefly describe the key idea of such a new curriculum before addressing the following two research questions of our empirical study:

a) Does the new curriculum lead to higher achievement gains than traditional approaches to teaching electricity in Germany?

b) Does the new curriculum lead to a better conceptual understanding of potential difference? 


\section{THE NEW CURRICULUM}

Taking into account the results from earlier studies on typical alternative conceptions in electricity [7] and the experience gained from a qualitative evaluation of an earlier version of the new curriculum in the form of one-to-one interviews [8], a new curriculum including appropriate course materials was developed. The objective of the new curriculum is to give students a qualitative, but robust, conception of voltage, current and resistance and to help them overcome typical alternative conceptions. It constantly endeavors to provide students with intuitive explanations that have their origins in everyday physical intuitions. In contrast to the CASTLE curriculum, it focuses on potential difference as the cause of an electric current from the very beginning. For this reason, the electric potential and potential differences are introduced even before the electric current by comparing the electric potential to air pressure. Another important difference to the CASTLE curriculum lies in the fact that the curriculum described here has been adapted to German school standards and, hence, places less emphasis on hands-on experiments with capacitors. Compared to traditional introductory courses to electricity in Germany, the most significant change of the new curriculum lies in the additional explicit introduction of the electric potential to explain voltage as a difference in electric potential. Although this may appear obvious as voltage is defined as potential difference, traditional approaches in Germany have tried to explain voltage as "energy per charge" or "the cause of current flow" without any reference to electric potential or potential differences. Not surprisingly, most students failed to develop an adequate conceptual understanding of voltage as a consequence. A comprehensive description of the new curriculum can be found in [8].

By building on students' everyday physical intuitions with air pressure, the new curriculum is in accordance with the ideas of diSessa [9], who interprets learning as the construction and reorganization of previously only loosely connected elements of knowledge, called "p-prims", into a coherent mental structure. These p-prims ("phenomenological primitives") represent naïve understandings of the physical world and are primitive in the sense that they represent minimal abstractions from everyday experience and that they only constitute a fragmented collection of ideas. In order to facilitate effective teaching, diSessa [10] proposes to build upon appropriate prior knowledge: "Students have a richness of conceptual resources to draw on. Attend to their ideas and help them build on the best of them" [pg. 41]. The pressure concept, as it is used here, is a good example for such a p-prim, as it only refers to the everyday experience that compressed air is "under pressure" and tries to push itself out of a container (e.g. an air mattress). In particular, the p-prim does not correspond to the refined physical scalar concept of pressure as a state variable that is difficult to understand for most young students. The curriculum then endeavors to embed this initially isolated p-prim into a cognitive structure by linking it to other p-prims such as "a pressure difference leads to an air flow". By using examples of everyday objects, students experience via hands-on experiments that air always flows from areas of high pressure to areas of low pressure and that pressure differences are the cause for an air flow. In addition, students learn that several layers of fabric reduce the air flow.

As a next step, the curriculum builds on these p-prims and tries to connect the familiar with the new. The key idea is that the intuitive air pressure concept, from the beginning, serves as a qualitative, low-abstraction prototype of the electric potential and potential difference in electric circuits. In the electron gas model, voltage as potential difference can hence be understood as an electric pressure difference across a resistor that is as much the cause for an electric current as air pressure differences are the cause for air flow. The air analogy is more compelling to learners than the water analogy, mainly because of the palpable compressibility of air and the fact that students gather experiences with it in everyday life (e.g. bicycle tires or air mattresses) [11]. For a discussion of the physics underlying the model, please refer to Chabay \& Sherwood [12].

\section{METHODS}

The quasi-experimental field study follows a pretestposttest-control-group design and is based on the testing of $\mathrm{N}=790$ students from Frankfurt/Main, Germany. The control group (CG), which was taught the traditional way by 11 teachers for an average of 23.5 lessons $(\mathrm{SD}=11.9)$, consisted of 17 junior high school classes and a total of $\mathrm{N}=357$ students. The slightly larger experimental group (EG), which was taught according to the new curriculum by 14 teachers for an average of 24.3 lessons $(\mathrm{SD}=9.8)$, consisted of 19 junior high school classes and a total of $\mathrm{N}=433$ students. The topic of electric circuits had not been covered before in any of the participating classes. In terms of group size and the amount of lessons taught, the CG and EG are, thus, comparable. However, for technical reasons, the participating teachers were not randomly assigned to the CG or EG, but had the choice of whether they wanted to teach traditionally or according to the new curriculum. From a psychometric perspective, this represents a shortcoming of the empirical study, because it cannot be ruled out that the differences between the CG and EG are in fact not attributable to the new curriculum, but to different levels of motivation, experience or aptitude on the teachers' side. 
In both groups, the same valid and reliable two-tier diagnostic multiple choice test was used for the pre- and posttest. The original test was constructed without reference to the new curriculum by an independent research group from Vienna and contained 22 items primarily on current and resistance, but not on voltage [13]. Therefore, in order to be able to evaluate students' understanding of voltage, we extended the original test by another four items on the concept. Since 22 out of the 26 items primarily dealt with current and resistance, the test used here is clearly unbiased towards the new curriculum that puts a particular emphasis on potential and potential difference. The advantage of the two-tier structure of the test is that students have to justify and explain their answers. This not only allows the identification of false-positive answers (i.e., correct answers despite an inadequate explanation), but more importantly, the identification of typical alternative conceptions about electric circuits. We counted an item as correct only if students gave both a correct answer and a correct explanation. The highest achievable score in the test, therefore, is 26 points.

Based on the data of the multiple choice test, the sample mean (M), standard error (SE) and statistical significance (p) are calculated for different aspects of both groups. Additionally, Cohen's d is reported, which is defined as the mean difference between two samples divided by the (pooled) standard deviation. Cohen's d represents an effect size and is a quantitative measure of whether an effect is meaningful within the research context. While a Cohen's d value between 0.5 and 0.8 represents a medium effect, a Cohen's d value larger than 0.8 indicates a large effect [14].

\section{RESULTS}

\section{A. Achievement gains}

A first analysis of the test results after instruction shows that students of the experimental group, on average, achieved a higher score in the posttest $(\mathrm{M}=12.99$, $\mathrm{SE}=0.27)$ than students of the control group $(\mathrm{M}=8.63$, $\mathrm{SE}=0.24)$. An unequal variances t-test shows that the difference between the two groups is highly significant $(t(787.60)=12.14, p<.001)$ and represents a medium-sized effect $\mathrm{d}=.85$.

As shown in Fig. 1, however, the mean test score before instruction was lower in the control group $(\mathrm{M}=5.09$, $\mathrm{SE}=0.15)$ than in the experimental group $(\mathrm{M}=6.29$, $\mathrm{SE}=0.16$ ). An unequal variances t-test shows that this difference in the pretest is highly significant $(\mathrm{t}(785.86)=5.41, \mathrm{p}<.001)$. It is therefore advisable to look at the difference between the pre- and posttest results in order to compare the net effect of instruction. On average, the absolute achievement gain by students who were taught according to the new curriculum $(\mathrm{M}=6.70, \mathrm{SE}=.26)$ is almost twice as high as that of traditionally taught students $(\mathrm{M}=3.54, \mathrm{SE}=.24)$. A mixed ANOVA with time of measurement (pretest, posttest) as a within-subjects factor and group (CG, EG) as between-subjects factor shows that the difference in absolute achievement gain between EG and $\mathrm{CG}$ is highly significant $(\mathrm{F}(1,788)=76.21, \mathrm{p}<.001)$ and represents a medium-sized effect $\mathrm{d}=.62$.

As the highest achievable score in the test is 26 points, a student with a high pretest result cannot achieve as high an absolute achievement gain as a student with a low pretest result (defined as the difference between the pre- and posttest). An alternate approach to compare the learning progress of students with different pretest results is to look at the relative achievement gain, which is defined as the absolute achievement gain divided by the highest possible achievement gain. In other words, the relative achievement gain is a measure for the achieved learning progress in relation to the learning progress that could have theoretically been achieved. In the experimental group the average relative achievement gain $(\mathrm{M}=34 \%, \mathrm{SE}=1 \%)$ is more than twice as high as in the control group $(\mathrm{M}=16 \%$, $\mathrm{SE}=1 \%$ ). An unequal variances t-test shows that the difference between the two groups is highly significant $(\mathrm{t}(787.73)=9.70, \mathrm{p}<.001)$.

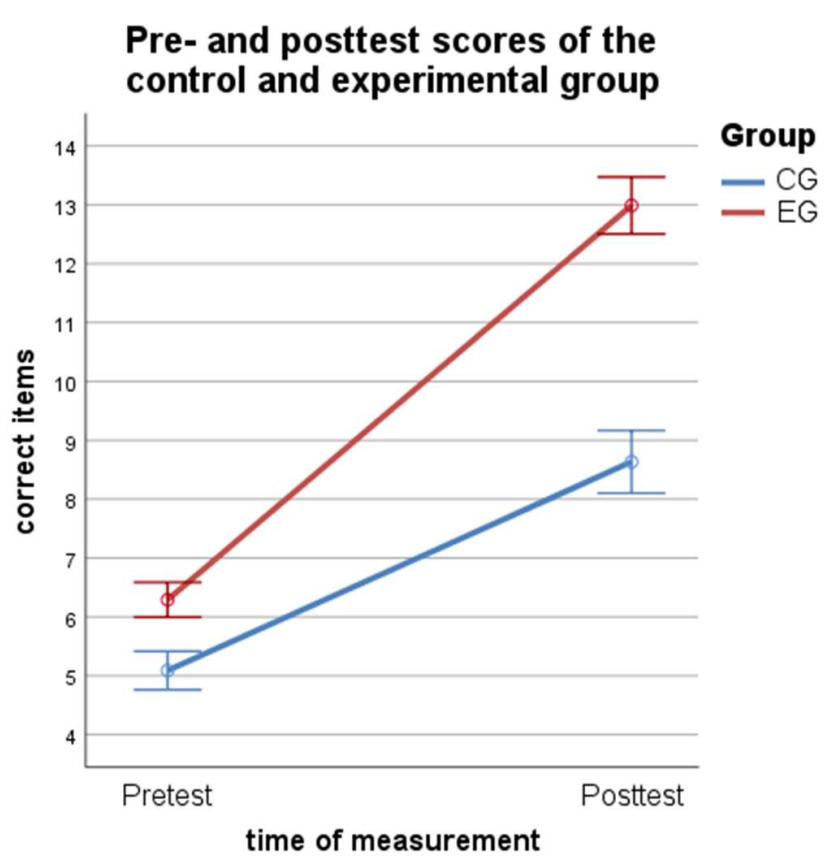

FIG 1. Pre- and posttest (raw) scores of the control and experimental group with $95 \%$ confidence intervals. The difference in pretest scores as well as posttest scores is highly significant $(\mathrm{p}<.001)$. 


\section{B. Conceptual understanding of voltage}

A comparison of students' conceptual understanding of voltage after instruction suggests that the new curriculum leads to a better understanding of voltage than the traditional approaches. On average, more students of the EG could answer at least one of the four items on voltage correctly $(\mathrm{M}=48 \%)$ than students of the $\mathrm{CG}(\mathrm{M}=12 \%)$, which represents a highly significant difference $\left(\chi^{2}(1)=114.44\right.$, $p<.001, \phi=.38)$. The new curriculum particularly appears to lead to a better understanding that voltage, in contrast to current, can only be measured between two points in a circuit because voltage refers to a difference in electric potential. Based on the answers given to three items on a closed circuit, $92 \%$ of the students in the CG had conceptual difficulties with this idea, compared to only $60 \%$ in the EG (see Fig. 2). This difference between the two groups is highly significant $\left(\chi^{2}(1)=54.36, \mathrm{p}<.001, \phi=.36\right)$. However, based on the answers given to an item on an open circuit, there seems to be no significant difference between the $\mathrm{EG}(\mathrm{M}=60 \%)$ and the $\mathrm{CG}(\mathrm{M}=54 \%)$ with regard to the widespread alternative conception that voltage is seen as a property of the electric current $\left(\chi^{2}(1)=1.28, \mathrm{p}=.26\right.$, $\phi=.05)$. Here, the majority of students of both groups wrongly concluded that if the current is zero, the voltage had to be zero too across all points in the open circuit including across the open switch.

\section{CONCLUSION \& FUTURE WORK}

The findings are promising in that the new curriculum appears to not only lead to achievement gains of about twice that of the control group, but also to a significantly better conceptual understanding of potential difference.

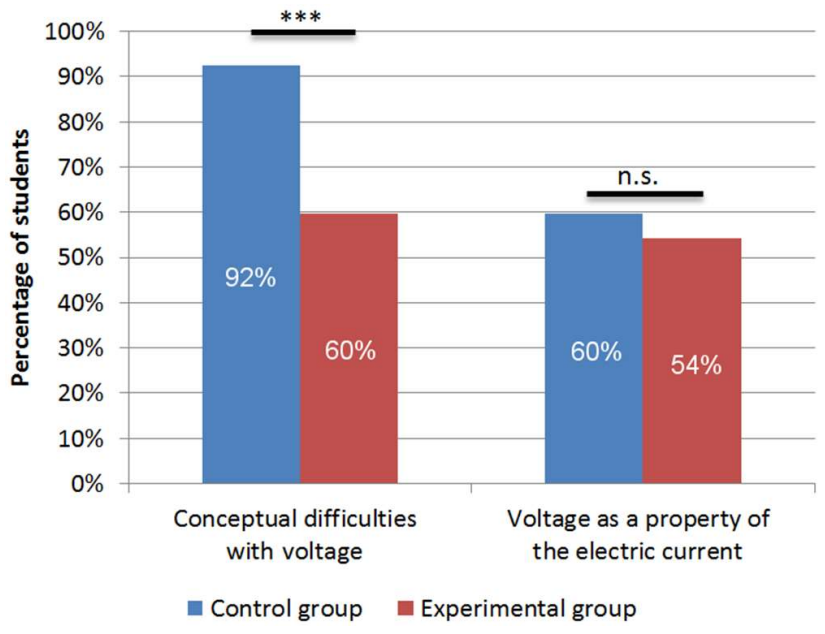

FIG 2. Conceptual understanding of voltage (the black lines indicate the significance level: $* * * \mathrm{p}<.001$; n.s. $\mathrm{p} \geq .05$ )

However, the fact that more than half of the students taught according to the new curriculum could not even answer one of the four items on voltage correctly, clearly demonstrates that voltage remains a difficult concept. Also, the air pressure analogy could have caused new alternative conceptions that were not covered by the test instrument used in this study. In a future study, it would therefore be interesting to identify these potential new alternative conceptions, e.g. by conducting one-to-one interviews with individual students. As the two groups differ in terms of their pretest results and as the clustered data structure (students are nested within classes) has been ignored so far, the use of more advanced statistical methods such as a multilevel analysis are planned for future investigations.
[1] R. Cohen, B. Eylon, and M. Ganiel, Am. J. Phys. 51, 5 (1983)

[2] R. Duit, W. Jung, and C.v. Rhöneck (Eds.), Aspects of Understanding Electricity - Proceedings of an International Workshop (Schmidt \& Klaunig, Kiel, 1985).

[3] C.v. Rhöneck, Naturwissenschaften im Unterricht Physik 34, 13 (1986).

[4] D.M. Shipstone, C.v. Rhöneck, W. Jung, C. Kärrqvist, J.-J. Dupin, S. Johsua, and P. Licht, Int J Sci Educ 10, 3 (1988).

[5] M.S. Steinberg et al. (2016). The CASTLE project. https://www.pasco.com/prodCompare/castle-kit/ retrieved 6/6/2017.

[6] M.S. Steinberg and C.L. Wainwright, Phys. Teach 31, 6 (1993).

[7] L.C. McDermott and P.S. Shaffer, Am. J. Phys. 60, 11 (1992).
[8] J.-P. Burde and T. Wilhelm, in Proceedings of ESERA, Helsinki, 2015, edited by J. Lavonen et al. (European Science Education Research Association, Helsinki, 2016).

[9] A. diSessa, Cogn Instr 10, 2-3 (1993).

[10] A. diSessa, in International Handbook of Research on Conceptual Change, edited by S. Vosniadou (Routledge, New York, 2008).

[11] J.-P. Burde and T. Wilhelm, Praxis der Naturwissenschaften - Physik in der Schule 65, 1 (2016).

[12] R.W. Chabay and B.A. Sherwood, Matter \& Interactions (Wiley, Hoboken N.Y., 2011), Vol. 1, p. 751.

[13] H. Urban-Woldron and M. Hopf, ZfDN 18 (2012).

[14] J. Cohen, Statistical Power Analysis for the Behavioral Sciences (Lawrence Erlbaum Associates, New York, 1988). 